\begin{tabular}{c|c|}
$\begin{array}{c}\text { SELECCIONES MATEMÁTICAS } \\
\text { Universidad Nacional de Trujillo } \\
\text { ISSN: } 2411-1783 \text { (Online) } \\
\text { Vol. 05(01): } 17-26(2018)\end{array}$ \\
\hline
\end{tabular}

\title{
Un Sistema De Boussinesq Completamente De Tipo-KdV en Espacios de Baja Regularidad
}

\author{
A completely KdV-type Boussinesq system in low regularity spaces \\ Juan Montealegre* and Zelideth Pérez ${ }^{\dagger}$
}

Received, Feb. 12, 2018

Accepted, Jun. 02, 2018

DOI: http://dx.doi.org/10.17268/sel.mat.2018.01.03

\section{Resumen}

En este artculo estudiamos la buena formulacin del problema de Cauchy para un sistema de Boussinesq formado por dos ecuaciones de Korteweg-de Vries acopladas a travs de la parte lineal y los trminos no lineales. Primero demostramos su buena formulacin local en los espacios de Sobolev $H^{s}(\mathbb{R}) \times H^{s}(\mathbb{R}), s>-3 / 4$, usando el estimado bilineal de Kenig, Ponce y Vega en los espacios de restriccin de la transformada de Fourier [4, 12]. Despus demostramos la buena formulacin global en $H^{s}(\mathbb{R}) \times H^{s}(\mathbb{R})$ para $s>-3 / 10$, nuestra prueba procede por el mtodo de las cantidades casi conservadas, a veces llamado el "mtodo-I" [5, 6].

Palabras clave. Problema de Cauchy, Ecuacin de Korteweg-de Vries, Buena formulacin global, Espacios de Bourgain, cantidades casi conservadas.

\begin{abstract}
In this paper we study the well-posedness of Cauchy problem for a Boussinesq system formed by two Kortewegde Vries equations coupled through the linear part and the non-linear terms. First we proof its local well-posedness in the Sobolev spaces $H^{s}(\mathbb{R}) \times H^{s}(\mathbb{R}), s>-3 / 4$, using the bilinear estimate established by Kenig, Ponce and Vega in the Fourier transform restriction spaces [4, 12]. After, we prove the global well-posedness in $H^{s}(\mathbb{R}) \times$ $H^{s}(\mathbb{R})$ for $s>-3 / 10$, our proof proceeds by the method of almost conservation laws, sometimes called the "I-method" [5, 6].
\end{abstract} laws.

Keywords. Cauchy problem, Korteweg-de Vries equations, Global well posedness, Bourgain spaces, almost conservation

1. Introduccin. En este artculo consideramos el problema de Cauchy para un sistema de Boussinesq formado por dos ecuaciones de Korteweg-de Vries acopladas a travs de la parte lineal y los trminos no lineales, de forma precisa, el problema es

$$
\left\{\begin{array}{l}
\partial_{t} w+\partial_{x} \eta+\partial_{x}^{3} \eta+\eta \partial_{x} \eta=0 \\
\partial_{t} \eta+\partial_{x} w+\partial_{x}^{3} w+\partial_{x}(w \eta)=0 \\
w(x, 0)=w_{0}(x), \quad \eta(x, 0)=\eta_{0}(x)
\end{array}\right.
$$

donde $w=w(x, t)$ y $\eta=\eta(x, t)$ son las funciones incgnitas, $(x, t) \in \mathbb{R}^{2}$, mientras que $w_{0}$ y $\eta_{0}$ son funciones dadas.

\footnotetext{
*Pontificia Universidad Catlica del Perú, Departamento de Ciencias. Av. Universitaria 1801, San Miguel, Lima 32, Perú (jmscottepucp.edu.pe).

$\dagger$ Universidad Nacional Agraria La Molina, Facultad de Ciencias. Av. La Molina s/n, La Molina, Lima 12, Perú' (zperez@lamolina.edu.pe).

This work is licensed under the Creative Commons Attribution-NoComercial-ShareAlike 4.0.
} 
El sistema en (1.1) es uno de los sistemas de Boussinesq descrito por Bona, Chen y Saut en [1, 2]. Las ecuaciones de Boussinesq, deducidas de las ecuaciones de Euler, modelan la propagacin de ondas, de pequea amplitud y longitud de onda larga sobre la superficie de un canal de fondo plano, estas ecuaciones son las $\mathrm{ms}$ simples de las que capturan los efectos dispersivos y no lineales de la onda. En el sistema (1.1) las variables adimensionales $\eta$ y $w$ representan respectivamente, la deflexin de la superficie libre del lquido respecto a su posicin de reposo y la velocidad horizontal del fluido a una profundidad de $\sqrt{\frac{2}{3}} h$, donde $h$ es la profundidad del fluido en reposo. Este modelo es un sistema de ecuaciones de Korteweg-de Vries acopladas mediante los efectos dispersivos y los trminos no lineales.

La buena formulacin local del problema (1.1) en los espacios $H^{s}(\mathbb{R}) \times H^{s}(\mathbb{R})$ para $s>\frac{3}{2}$ se demostr en [15], utilizando el mtodo de regularizacin parablica [8,9] y los estimados de Bona-Smith [3]. En el artculo [2] se demostr la buena formulacin local $H^{s}(\mathbb{R}) \times H^{s}(\mathbb{R})$ con $s>\frac{3}{4}$, siguiendo las ideas desarrolladas en [10, 11].

En [4] Bourgain desarroll un mtodo del anlisis armnico para resolver ecuaciones de evolucin no lineales. La parte esencial consiste en elegir adecuadamente un espacio de funciones cuyas normas se definen por la transformada de Fourier en las variables espacio-tiempo e involucra la estructura especfica de la parte lineal de la ecuacin. Usando este mtodo Bourgain prob que el problema asociado a la ecuacin de Korteweg-de Vries es localmente bien formulado cuando la informacin inicial $w_{0}$ pertenece al espacio de Sobolev $H^{s}(\mathbb{R})$ con $s \geq 0$.

Despus, Kenig, Ponce y Vega [12] mejoraron el resultado de Bourgain demostrando la buena formulacin local del problema asociado a la ecuacin de $\mathrm{KdV}$ para datos iniciales en $H^{s}(\mathbb{R})$ con $s>-3 / 4$. Siguiendo las ideas desarrolladas en [4] y [12], en este artculo demostraremos que el problema de Cauchy (1.1) es bien formulado localmente en los espacios $H^{s}(\mathbb{R}) \times H^{s}(\mathbb{R})$ con $s>-3 / 4$.

El segundo objetivo de este artculo consiste en demostrar la buena formulacin global en espacios de Sobolev. Como no hay cantidades conservadas para conseguir un estimado a priori en los espacios de Sobolev de ndices negativos, la buena formulacin global en estos espacios debe ser abordada con otras tcnicas. Uno de estos mtodos es el de las cantidades casi conservadas o tambin llamado el mtodo- $I$, presentado por Colliander, Keel, Staffilani, Takaoka y Tao [5, 6], que describiremos despus. Este es el mtodo que emplearemos para extender el resultado local a $H^{s}(\mathbb{R}) \times H^{s}(\mathbb{R})$ con $s>-3 / 10$, la solucin en cualquier intervalo de tiempo $[0, T]$ se obtendr de la solucin local por medio de un proceso iterativo en un nmero finito de pasos.

2. Transformacin del sistema y resultados principales. El problema de Cauchy (1.1) queda desacoplado en la parte lineal por medio del cambio de variables

$$
\left\{\begin{array}{l}
w=f+g \\
\eta=f-g
\end{array}\right.
$$

obtenindose as el problema equivalente,

$$
\left\{\begin{array}{l}
\partial_{t} f+\partial_{x} f+\partial_{x}^{3} f+\partial_{x} F(f, g)=0 \\
\partial_{t} g-\partial_{x} g-\partial_{x}^{3} g+\partial_{x} F(g, f)=0 \\
f(x, 0)=\frac{1}{2}\left[w_{0}(x)+\eta_{0}(x)\right] \\
g(x, 0)=\frac{1}{2}\left[w_{0}(x)-\eta_{0}(x)\right]
\end{array}\right.
$$

donde

$$
F(f, g)=\frac{3}{4} f^{2}-\frac{1}{2} f g-\frac{1}{4} g^{2} .
$$

Entonces, con un cambio de escala y un cambio de variables independientes obtenemos el problema de Cauchy equivalente

$$
\left\{\begin{array}{l}
\partial_{t} u+\partial_{x}^{3} u+\partial_{x} F(u, v)=0 \\
\partial_{t} v+\partial_{x}^{3} v+\partial_{x} F(v, u)=0 \\
u(x, 0)=u_{0}(x) \\
v(x, 0)=v_{0}(x)
\end{array}\right.
$$

donde $u_{0}(x)=\frac{1}{2}\left[w_{0}(x)+\eta_{0}(x)\right]$ y $v_{0}(x)=\frac{1}{2}\left[w_{0}(-x)-\eta_{0}(-x)\right]$.

Notemos que (2.1) tiene la estructura de dos ecuaciones de $\mathrm{KdV}$ acopladas solamente en los trminos no lineales. De los resultados de buena formulacin para (2.1) es fcil obtener los correspondientes resultados para (1.1).

El espacio de funciones donde vamos a encontrar solucin al problema (1.1) es el espacio de Bourgain denotado por $X_{s, b}$ con $s, b \in \mathbb{R}$, el cual es el subconjunto de $\mathcal{S}^{\prime}\left(\mathbb{R}^{2}\right)$ definido por

$$
X_{s, b}=\left\{u \in \mathcal{S}^{\prime}\left(\mathbb{R}^{2}\right):\|u\|_{s, b}^{2}=\int_{\mathbb{R}}\left\langle\tau-\xi^{3}\right\rangle^{2 b}\langle\xi\rangle^{2 s}|\widehat{u}(\xi, t)|^{2} d \xi d \tau<\infty\right\}
$$


donde $\langle\cdot\rangle=1+|\cdot|$ y $\widehat{u}$ denota la transformada de Fourier de $u$ en las variables $x$ y $t$, esto es

$$
\widehat{u}(\xi, \tau)=\frac{1}{\sqrt{2 \pi}} \int_{\mathbb{R}^{2}} e^{-i(x \xi+t \tau)} u(x, t) d x d t .
$$

Observamos que [13, Captulo 7]: el espacio de Schwartz $\mathcal{S}\left(\mathbb{R}^{2}\right)$ es denso en $X_{s, b}$ cualquiera que sean $s, b \in \mathbb{R}$ y si $b>1 / 2$, entonces $X_{s, b}$ est contenido continuamente en $C_{b}\left(\mathbb{R}_{t}: H_{x}^{s}(\mathbb{R})\right)$, el espacio de las funciones continuas y acotadas con la norma de $H_{x}^{s}(\mathbb{R})$.

Ahora establecemos los resultados principales de este artculo. El primer resultado trata sobre la buena formulacin local del problema (2.1) y se enuncia como sigue.

Teorema 2.1. Para cada $\left(u_{0}, v_{0}\right) \in H^{s}(\mathbb{R}) \times H^{s}(\mathbb{R}), s>-3 / 4$ yb $\left.\in\right] 1 / 2,1\left[\right.$ existe $T=T\left(\left\|u_{0}\right\|_{H^{s}},\left\|v_{0}\right\|_{H^{s}}\right)$ y una solucin nica de (2.1) en el intervalo de tiempo $[-T, T]$ que satisface

$$
\begin{gathered}
u, v \in C\left([-T, T]: H^{s}(\mathbb{R})\right), \\
u, v \in X_{s, b} \subseteq L_{x, l o c}^{p}\left(\mathbb{R}: L_{t}^{2}(\mathbb{R})\right), 1 \leq p \leq \infty, \\
\partial_{x} u^{2}, \partial_{x} v^{2} \in X_{s, b-1}, \\
\partial_{t} u, \partial_{t} v \in X_{s-3, b-1} .
\end{gathered}
$$

Nuestro prximo teorema trata sobre la buena formulacin global de (2.1). Ms precisamente, probaremos el siguiente resultado.

Teorema 2.2. El problema de Cauchy (2.1) es globalmente bien formulado en $H^{s}(\mathbb{R}) \times H^{s}(\mathbb{R})$, $s>-3 / 10$.

3. Resultados preliminares. Usando el principio de Duhamel [7, Captulo 2], obtenemos el sistema de ecuaciones integrales equivalente al problema (2.1),

$$
\left\{\begin{array}{l}
u(t)=W(t) u_{0}-\int_{0}^{t} W(t-\tau) \partial_{x} F(u(\tau), v(\tau)) d \tau \\
v(t)=W(t) v_{0}-\int_{0}^{t} W(t-\tau) \partial_{x} F(v(\tau), u(\tau)) d \tau
\end{array}\right.
$$

donde $\{W(t)\}_{t \in \mathbb{R}}$ es el grupo unitario asociado con el problema lineal definido por (2.1).

Para encontrar una solucin local para (2.1) reemplazamos (3.1) con el siguiente sistema de ecuaciones integrales,

$$
\left\{\begin{array}{l}
u(t)=\psi(t) W(t) u_{0}-\psi(t) \int_{0}^{t} W(t-\tau) \partial_{x} F(u(\tau), v(\tau)) d \tau \\
v(t)=\psi(t) W(t) v_{0}-\psi(t) \int_{0}^{t} W(t-\tau) \partial_{x} F(v(\tau), u(\tau)) d \tau
\end{array}\right.
$$

donde $\psi \in C_{0}^{\infty}(\mathbb{R}), 0 \leq \psi \leq 1$ es una funcin de truncamiento dada por,

$$
\psi(t)= \begin{cases}1 & ,|t|<1 \\ 0 & ,|t| \geq 2\end{cases}
$$

y $\psi_{\delta}(t)=\psi\left(\frac{t}{\delta}\right), 0<\delta \leq 1$.

Proposicin 3.1 (del estimado bilineal). Si $s>-3 / 4$, existe $b \in] 1 / 2,1[$ tal que

$$
\left\|\partial_{x}(u v)\right\|_{X_{s, b-1}} \leq C\|u\|_{X_{s, b}}\|v\|_{X_{s, b}} .
$$

Proposicin 3.2. Sean $\left.s \in \mathbb{R}, b, b^{\prime} \in\right] 1 / 2,1\left[\right.$ con $\left.b^{\prime}<b y \delta \in\right] 0,1[$, entonces,

$$
\begin{gathered}
\left\|\psi_{\delta}(t) W(t) \varphi\right\|_{X_{s, b}} \leq C \delta^{\frac{1-2 b}{2}}\|\varphi\|_{H^{s}} \\
\left\|\psi_{\delta}(t) F\right\|_{X_{s, b-1}} \leq C \delta^{\frac{b-b^{\prime}}{8\left(1-b^{\prime}\right)}}\|F\|_{X_{s, b^{\prime}-1}} \\
\left\|\psi_{\delta}(t) \int_{0}^{t} W(t-\tau) F(\tau) d \tau\right\|_{X_{s, b}} \leq C \delta^{\frac{1-2 b}{2}}\|F\|_{X_{s, b-1}}
\end{gathered}
$$




$$
\left\|\psi_{\delta}(t) \int_{0}^{t} W(t-\tau) F(\tau) d \tau\right\|_{H^{s}} \leq C \delta^{\frac{1-2 b}{2}}\|F\|_{X_{s, b-1}} .
$$

Las demostraciones de las proposiciones 3.1 y 3.2 se pueden ver en [12] y [14], respectivamente.

Para extender la solucin local a una global, definiremos el multiplicador de Fourier $I$ por,

$$
\widehat{I u}(\xi)=m(\xi) \widehat{u}(\xi),
$$

donde $m(\xi)$ es una funcin suave y montona dada por

$$
m(\xi)= \begin{cases}1 & \text { si }|\xi| \leq N \\ N^{-s}|\xi|^{s} & \text { si }|\xi|>2 N\end{cases}
$$

con $N \gg 1$ a ser fijado posteriormente.

Notemos que el operador $I$ es el operador identidad para las frecuencias bajas, es decir $\{\xi \in \mathbb{R}:|\xi|<N\}$ y un operador integral en las frecuencias altas. En general, $I: H^{s}(\mathbb{R}) \rightarrow L^{2}(\mathbb{R})$ es un operador acotado que conmuta con operadores diferenciables y

$$
\|u\|_{H^{s}} \leq\|I u\|_{L^{2}} \leq C N^{-s}\|u\|_{H^{s}} .
$$

Proposicin 3.3. Si $s>-\frac{3}{4}$, para cada $\frac{1}{2}<b<1$ existe $C$ tal que

$$
\left\|\partial_{x} I(u v)\right\|_{X_{s, b-1}} \leq C\|I u\|_{X_{s, b}}\|I v\|_{X_{s, b}} .
$$

Para probar la desigualdad en (3.11) se aplica (3.3) combinado con el estimado bilineal con regularidad extra siguiente.

Proposicin 3.4. Si $s>-\frac{3}{4}$, para cada $\frac{1}{2}<b<1$ existe $C$ independiente de $N$ se cumple que

$$
\left\|\partial_{x}[I(u v)-I u I v]\right\|_{X_{s, b-1}} \leq C N^{-\alpha}\|I u\|_{X_{s, b}}\|I v\|_{X_{s, b}} .
$$

Para la demostracin de las proposicin 3.4 se puede consultar la segunda seccin de [5].

Proposicin 3.5. Las siguientes igualdades se cumplen,

$$
\left\langle\partial_{x}(I u)^{2}, I u\right\rangle_{L^{2}}=0 \quad y \quad\left\langle\partial_{x}(I v)^{2}, I v\right\rangle_{L^{2}}=0
$$

$y$

$$
\frac{1}{2} I_{1}+I_{2}+\frac{1}{2} I_{3}+I_{4}=0
$$

donde

$$
\begin{array}{ll}
I_{1}=\int_{0}^{\delta}\left\langle\partial_{x}(I v)^{2}, I u\right\rangle_{L^{2}} d t & I_{2}=\int_{0}^{\delta}\left\langle\partial_{x}(I u I v), I u\right\rangle_{L^{2}} d t \\
I_{3}=\int_{0}^{\delta}\left\langle\partial_{x}(I u)^{2}, I v\right\rangle_{L^{2}} d t & I_{4}=\int_{0}^{\delta}\left\langle\partial_{x}(I u I v), I v\right\rangle_{L^{2}} d t .
\end{array}
$$

Demostracin: La prueba de (3.13) es trivial. Adems,

$$
I_{2}=-\int_{0}^{\delta}\left\langle I u I v, \partial_{x} I u\right\rangle_{L^{2}} d t=-\int_{0}^{\delta}\left\langle I v, I u \partial_{x} I u\right\rangle_{L^{2}} d t=-\frac{1}{2} \int_{0}^{\delta}\left\langle I v, \partial_{x}(I u)^{2}\right\rangle_{L^{2}} d t=-\frac{1}{2} I_{3}
$$

$\mathrm{y}$

$$
I_{4}=-\int_{0}^{\delta}\left\langle I u I v, \partial_{x} I v\right\rangle_{L^{2}} d t=-\int_{0}^{\delta}\left\langle I u, I v \partial_{x} I v\right\rangle_{L^{2}} d t=-\frac{1}{2} \int_{0}^{\delta}\left\langle I u, \partial_{x}(I v)^{2}\right\rangle_{L^{2}} d t=-\frac{1}{2} I_{1},
$$

entonces

$$
\frac{1}{2} I_{1}+I_{2}+\frac{1}{2} I_{3}+I_{4}=\frac{1}{2} I_{1}+\left(-\frac{1}{2} I_{3}\right)+\frac{1}{2} I_{3}+\left(-\frac{1}{2} I_{1}\right)=0
$$


4. Demostracin del teorema 2.1. Consideremos el siguiente espacio de funciones en donde buscamos una solucin para el problema de Cauchy (2.1). Dado $\left(u_{0}, v_{0}\right) \in H^{s} \times H^{s}, s>-3 / 4$ y $b>1 / 2$, definimos

$$
\mathcal{E}_{s, b, R}=\left\{(u, v) \in \mathbb{X}_{s, b}:\|(u, v)\|_{\mathbb{X}_{s, b}} \leq R\right\}
$$

donde $\mathbb{X}_{s, b}=X_{s, b} \times X_{s, b} \mathrm{y}$

$$
\|(u, v)\|_{\mathbb{X}_{s, b}}=\|u\|_{X_{s, b}}+\|v\|_{X_{s, b}} .
$$

Entonces $\mathcal{E}_{s, b, R}$ es un espacio mtrico completo.

Para $(u, v) \in \mathcal{E}_{s, b, R}$ definimos $\Gamma(u, v)=\left(\Gamma_{1}(u, v), \Gamma_{2}(u, v)\right)$ donde las aplicaciones $\Gamma_{1} \mathrm{y} \Gamma_{2}$ son definidos por

$$
\left\{\begin{array}{l}
\Gamma_{1}(u, v)(t)=\psi(t) W(t) u_{0}-\psi(t) \int_{0}^{t} W(t-\tau) \psi_{\delta}(\tau) \partial_{x} F(u(\tau), v(\tau)) d \tau \\
\Gamma_{2}(u, v)(t)=\psi(t) W(t)-\psi(t) \int_{0}^{t} W(t-\tau) \psi_{\delta}(\tau) \partial_{x} F(v(\tau), u(\tau)) d \tau
\end{array}\right.
$$

La buena definicin de $\Gamma$ es garantizada por las proposiciones 3.1 y 3.2. Probaremos que existe $C>0$ y $R>0$ tal que $\Gamma$ aplica $\mathcal{E}_{s, b, R}$ en s mismo y es una contraccin.

Usando la desigualdad triangular, (3.4), (3.6) y (3.5) con $b^{\prime}<b$ conseguimos de (4.1),

$$
\begin{aligned}
\left\|\Gamma_{1}(u, v)\right\|_{X_{s, b}} & \leq\left\|\psi(t) W(t) u_{0}\right\|_{X_{s, b}}+\left\|\psi(t) \int_{0}^{t} W(t-\tau) \psi_{\delta}(\tau) \partial_{x} F(u(\tau), v(\tau)) d \tau\right\|_{X_{s, b}} \\
& \leq C\left\|u_{0}\right\|_{H^{s}}+C\left\|\psi_{\delta}(\cdot) \partial_{x} F(u, v)\right\|_{X_{s, b-1}} \\
& \leq C\left\|u_{0}\right\|_{H^{s}}+C \delta^{\frac{b-b^{\prime}}{8\left(1-b^{\prime}\right)}}\left\|\partial_{x} F(u, v)\right\|_{X_{s, b^{\prime}-1}} \\
& \leq C\left\|u_{0}\right\|_{H^{s}}+C \delta^{\frac{b-b^{\prime}}{8\left(1-b^{\prime}\right)}}\left(\left\|\partial_{x} u^{2}\right\|_{X_{s, b^{\prime}-1}}+\left\|\partial_{x}(u v)\right\|_{X_{s, b^{\prime}-1}}+\left\|\partial_{x} v^{2}\right\|_{X_{s, b^{\prime}-1}}\right) .
\end{aligned}
$$

Pero (3.3) y la desigualdad $\|\cdot\|_{X_{s, b^{\prime}}} \leq\|\cdot\|_{X_{s, b}}$ implican,

$$
\begin{aligned}
\left\|\Gamma_{1}(u, v)\right\|_{X_{s, b}} & \leq C_{1}\left\|u_{0}\right\|_{H^{s}}+C_{2} \delta^{\frac{b-b^{\prime}}{8\left(1-b^{\prime}\right)}}\left(\|u\|_{X_{s, b^{\prime}}}^{2}+\|u\|_{X_{s, b^{\prime}}}\|v\|_{X_{s, b^{\prime}}}+\|v\|_{X_{s, b^{\prime}}}^{2}\right) \\
& \leq C_{1}\left\|u_{0}\right\|_{H^{s}}+C_{2} \delta^{\frac{b-b^{\prime}}{8\left(1-b^{\prime}\right)}}\left(\|u\|_{X_{s, b}}+\|v\|_{X_{s, b}}\right)^{2} \\
& =C_{1}\left\|\left(u_{0}, v_{0}\right)\right\|_{\mathbb{X}_{s, b}}+C_{2} \delta^{\frac{b-b^{\prime}}{8\left(1-b^{\prime}\right)}}\|(u, v)\|_{\mathbb{X}_{s, b}}^{2} .
\end{aligned}
$$

Elegimos $\left\|\left(u_{0}, v_{0}\right)\right\|_{\mathbb{X}_{s, b}} \leq R / 4 C_{1}$ y $\delta>0$ tal que $\delta^{\frac{b-b^{\prime}}{8\left(1-b^{\prime}\right)}} \leq 1 / 4 C_{2} R$, entonces

$$
\left\|\Gamma_{1}(u, v)\right\|_{X_{s, b}} \leq C_{1}\left(\frac{R}{4 C_{1}}\right)+C_{2}\left(\frac{1}{4 C_{2} R}\right) R^{2}=\frac{R}{2} .
$$

De la misma manera obtenemos $\left\|\Gamma_{2}(u, v)\right\|_{X_{s, b}} \leq R / 2$, por lo tanto

$$
\|\Gamma(u, v)\|_{\mathbb{X}_{s, b}}=\left\|\Gamma_{1}(u, v)\right\|_{X_{s, b}}+\left\|\Gamma_{2}(u, v)\right\|_{X_{s, b}} \leq R
$$

y $\Gamma(u, v) \in \mathcal{E}_{s, b, R}$.

Ahora necesitamos mostrar que $\Gamma$ es una contraccin. Para esto, si $(u, v),\left(u_{1}, v_{1}\right) \in \mathcal{E}_{s, b, R}$ de $(4.1)$ y (3.6)

$$
\begin{aligned}
&\left\|\Gamma_{1}(u, v)-\Gamma_{1}\left(u_{1}, v_{1}\right)\right\|_{X_{s, b}} \\
& \leq\left\|\psi(t) \int_{0}^{t} W(t-\tau) \psi_{\delta}(\tau)\left[\partial_{x} F(u(\tau), v(\tau))-\partial_{x} F\left(u_{1}(\tau), v_{1}(\tau)\right)\right] d \tau\right\|_{X_{s, b}} \\
& \leq C\left\|\psi_{\delta}(\cdot)\left[\partial_{x} F(u, v)-\partial_{x} F\left(u_{1}, v_{1}\right)\right]\right\|_{X_{s, b-1}},
\end{aligned}
$$

pero

$$
\begin{aligned}
\partial_{x} F(u, v)-\partial_{x} F\left(u_{1}, v_{1}\right)= & \partial_{x}\left[\frac{3}{4}\left(u^{2}-u_{1}^{2}\right)-\frac{1}{2}\left(u v-u_{1} v_{1}\right)-\frac{1}{4} v^{2}+\frac{1}{4} v_{1}^{2}\right] \\
= & \frac{3}{4} \partial_{x}\left[\left(u+u_{1}\right)\left(u-u_{1}\right)\right]-\frac{1}{2} \partial_{x}\left[u\left(v-v_{1}\right)\right] \\
& +\frac{1}{2} \partial_{x}\left[v_{1}\left(u-u_{1}\right)\right]-\frac{1}{4} \partial_{x}\left[\left(v+v_{1}\right)\left(v-v_{1}\right)\right],
\end{aligned}
$$


entonces, por la desigualdad triangular, (3.5) con $b^{\prime}<b$, (3.3) y la desigualdad $\|\cdot\|_{X_{s, b^{\prime}}} \leq\|\cdot\|_{X_{s, b}}$, obtenemos

$$
\begin{aligned}
&\left\|\Gamma_{1}(u, v)-\Gamma_{1}\left(u_{1}, v_{1}\right)\right\|_{X_{s, b}} \leq C \delta^{\frac{b-b^{\prime}}{8\left(1-b^{\prime}\right)}}\left[\left\|\partial_{x}\left[\left(u+u_{1}\right)\left(u-u_{1}\right)\right]\right\|_{X_{s, b^{\prime}-1}}+\left\|\partial_{x}\left[u\left(v-v_{1}\right)\right]\right\|_{X_{s, b^{\prime}-1}}\right. \\
&\left.+\left\|\partial_{x}\left[v_{1}\left(u-u_{1}\right)\right]\right\|_{X_{s, b^{\prime}-1}}+\left\|\partial_{x}\left[\left(v+v_{1}\right)\left(v-v_{1}\right)\right]\right\|_{X_{s, b^{\prime}-1}}\right] \\
& \leq C \delta^{\frac{b-b^{\prime}}{8\left(1-b^{\prime}\right)}}\left[\left\|u+u_{1}\right\|_{X_{s, b}}\left\|u-u_{1}\right\|_{X_{s, b}}+\|u\|_{X_{s, b}}\left\|v-v_{1}\right\|_{X_{s, b}}\right. \\
&\left.\quad+\left\|v_{1}\right\|_{X_{s, b}}\left\|u-u_{1}\right\|_{X_{s, b}}+\left\|v+v_{1}\right\|_{X_{s, b}}\left\|v-v_{1}\right\|_{X_{s, b}}\right] .
\end{aligned}
$$

Luego,

$$
\begin{aligned}
\left\|\Gamma_{1}(u, v)-\Gamma_{1}\left(u_{1}, v_{1}\right)\right\|_{X_{s, b} \leq} & C \delta^{\frac{b-b^{\prime}}{8\left(1-b^{\prime}\right)}}\left[\left(\|u\|_{X_{s, b}}+\left\|u_{1}\right\|_{X_{s, b}}+\left\|v_{1}\right\|_{X_{s, b}}\right)\left\|u-u_{1}\right\|_{X_{s, b}}\right. \\
& \left.+\left(\|u\|_{X_{s, b}}+\|v\|_{X_{s, b}}+\left\|v_{1}\right\|_{X_{s, b}}\right)\left\|v-v_{1}\right\|_{X_{s, b}}\right] \\
\leq & 3 C \delta^{\frac{b-b^{\prime}}{8\left(1-b^{\prime}\right)}} R\left(\left\|u-u_{1}\right\|_{X_{s, b}}+\left\|v-v_{1}\right\|_{X_{s, b}}\right) \\
\leq & 3 C \delta^{\frac{b-b^{\prime}}{8\left(1-b^{\prime}\right)}} R\left\|\left(u-u_{1}, v-v_{1}\right)\right\|_{\mathbb{X}_{s, b}}
\end{aligned}
$$

De la misma manera obtenemos

$$
\left\|\Gamma_{2}(u, v)-\Gamma_{2}\left(u_{1}, v_{1}\right)\right\|_{X_{s, b}} \leq 3 C \delta^{\frac{b-b^{\prime}}{8\left(1-b^{\prime}\right)}} R\left\|\left(u-u_{1}, v-v_{1}\right)\right\|_{\mathbb{X}_{s, b}} .
$$

Entonces

$$
\left\|\left(\Gamma_{1}(u, v)-\Gamma_{1}\left(u_{1}, v_{1}\right), \Gamma_{2}(u, v)-\Gamma_{2}\left(u_{1}, v_{1}\right)\right)\right\|_{\mathbb{X}_{s, b}} \leq 6 C \delta^{\frac{b-b^{\prime}}{8\left(1-b^{\prime}\right)}} R\left\|\left(u-u_{1}, v-v_{1}\right)\right\|_{\mathbb{X}_{s, b}},
$$

si elegimos $\delta>0$ tal que $6 C \delta^{\frac{b-b^{\prime}}{8\left(1-b^{\prime}\right)}} R<1$, $\Gamma$ es una contraccin.

Por el teorema del punto fijo de Banach existe exactamente un punto fijo de $\Gamma$ en $\mathcal{E}_{s, b, R}$. Por un argumento bien conocido la unicidad de la solucin $(u, v)$ se obtiene tambin en $\mathbb{X}_{s, b}$. Adems, es fcil ver que la aplicacin

$$
\Lambda_{T}: \mathbb{X}_{s, b} \times \mathcal{B}_{R} \longrightarrow \mathbb{X}_{s, b}
$$

definida por

$$
\Lambda_{T}\left((u, v),\left(u_{0}, v_{0}\right)\right)=\left(\Psi_{u_{0}}(u, v), \Psi_{v_{0}}(u, v)\right)
$$

es analtica, por lo tanto, el teorema de la funcin implcita implica la analiticidad de la aplicacin del flujo $\Theta$ : $\left(u_{0}, v_{0}\right) \in \mathcal{B}_{R} \mapsto(u, v) \in C\left([0, T]: H^{s}\right) \times C\left([0, T]: H^{s}\right)$.

5. Demostracin del teorema 2.2. Esta seccin es dedicada a extender la solucin local obtenida en la seccin anterior a la solucin global. Usando las leyes de conservacin habituales tenemos solucin global en $H^{s}(\mathbb{R}) \times H^{s}(\mathbb{R})$, $s \geq 0$. Por lo tanto, a lo largo de esta seccin suponemos $s<0$. Nuestro objetivo aqu es derivar una cantidad casi conservada y usarla para probar el teorema 2.2.

Tenemos la siguiente variante de la buena formulacin local del problema de Cauchy (2.1).

Proposicin 5.1. Para cada $\left(u_{0}, v_{0}\right) \in H^{s}(\mathbb{R}) \times H^{s}(\mathbb{R}), s>-\frac{3}{4}$, el problema de Cauchy (2.1) es localmente bien formulado en el espacio de Banach $I^{-1} L^{2} \times I^{-1} L^{2}$ dotado de la norma $\|\mid(u, v)\|\|=\| I u\left\|_{L^{2}}+\right\| I v \|_{L^{2}}$, con el tiempo de existencia $T$ que cumple

$$
\left(\left\|I u_{0}\right\|_{L^{2}}+\left\|I v_{0}\right\|_{L^{2}}\right)^{-\alpha} \leq C T, \quad \alpha>0 .
$$

Adems,

$$
\left\{\begin{array}{l}
\|\psi(\cdot / T) I u\|_{X_{0, b}} \leq C\left\|I u_{0}\right\|_{L^{2}} \\
\|\psi(\cdot / T) I v\|_{X_{0, b}} \leq C\left\|I v_{0}\right\|_{L^{2}} .
\end{array}\right.
$$

La demostracin de esta proposicin se obtiene con el mismo procedimiento usado para probar la buena formulacin local del problema (2.1) desde que se dispone del estimado bilineal de la proposicin 3.3. 
Ahora procedemos a calcular la cantidad casi conservada. Cuando aplicamos el operador $I$ al sistema (2.1), obtenemos el siguiente problema de Cauchy

$$
\left\{\begin{array}{l}
\partial_{t} I u+\partial_{x}^{3} I u+\partial_{x}\left(\frac{3}{4} I u^{2}-\frac{1}{2} I(u v)-\frac{1}{4} I v^{2}\right)=0 \\
\partial_{t} I v+\partial_{x}^{3} I v+\partial_{x}\left(\frac{3}{4} I v^{2}-\frac{1}{2} I(u v)-\frac{1}{4} I u^{2}\right)=0 \\
I u(0)=I u_{0}(x) \\
I v(0)=I v_{0}(x)
\end{array}\right.
$$

Entonces, usando integracin por partes, conseguimos

$$
\begin{aligned}
\frac{d}{d t}\|I u(t)\|_{L^{2}}^{2} & =2\left\langle\frac{d}{d t} I u(t), I u(t)\right\rangle_{L^{2}} \\
& =2\left\langle-\partial_{x}^{3} I u-\partial_{x}\left(\frac{3}{4} I u^{2}-\frac{1}{2} I(u v)-\frac{1}{4} I v^{2}\right), I u\right\rangle_{L^{2}} \\
& =-2\left\langle\partial_{x}^{3} I u, I u\right\rangle_{L^{2}} 2\left\langle\partial_{x}\left(-\frac{3}{4} I u^{2}+\frac{1}{2} I(u v)+\frac{1}{4} I v^{2}\right), I u\right\rangle_{L^{2}} \\
& =\left\langle\partial_{x}\left(-\frac{3}{2} I u^{2}+I(u v)+\frac{1}{2} I v^{2}\right), I u\right\rangle_{L^{2}},
\end{aligned}
$$

pues se tiene que $\left\langle\partial_{x}^{3} I u, I u\right\rangle=0$. Entonces, integrando respecto de $t$ en $[0, \delta]$ obtenemos

$$
\begin{aligned}
\|I u(\delta)\|_{L^{2}}^{2} & =\|I u(0)\|_{L^{2}}^{2}+\int_{0}^{\delta} \frac{d}{d t}\|I u(t)\|_{L^{2}}^{2} d t \\
& =\left\|I u_{0}\right\|_{L^{2}}^{2}-\int_{0}^{\delta}\left\langle\partial_{x}\left(\frac{3}{2} I u^{2}(t)-I(u v)(t)-\frac{1}{2} I v^{2}(t)\right), I u(t)\right\rangle_{L^{2}} d t \\
& =\left\|I u_{0}\right\|_{L^{2}}^{2}+R_{1}(\delta)
\end{aligned}
$$

donde

$$
R_{1}(\delta)=-\int_{0}^{\delta}\left\langle\partial_{x}\left(\frac{3}{2} I u^{2}(t)-I(u v)(t)-\frac{1}{2} I v^{2}(t)\right), I u(t)\right\rangle_{L^{2}} d t
$$

De la misma forma

$$
\|I v(\delta)\|_{L^{2}}^{2}=\left\|I v_{0}\right\|_{L^{2}}^{2}+R_{2}(\delta),
$$

donde

$$
R_{2}(\delta)=-\int_{0}^{\delta}\left\langle\partial_{x}\left(\frac{3}{2} I v^{2}-I(u v)-\frac{1}{2} I u^{2}\right), I v\right\rangle_{L^{2}} d t .
$$

Definimos $R(\delta):=R_{1}(\delta)+R_{2}(\delta)$, as tenemos de (5.4) y (5.6),

$$
\|I u(\delta)\|_{L^{2}}^{2}+\|I v(\delta)\|_{L^{2}}^{2}=\left\|I u_{0}\right\|_{L^{2}}^{2}+\left\|I v_{0}\right\|_{L^{2}}^{2}+R(\delta) .
$$

Usando la proposicin 3.5 tenemos

$$
\begin{aligned}
R(\delta)= & -\frac{3}{2} \int_{0}^{\delta}\left\langle\partial_{x} I u^{2}, I u\right\rangle_{L^{2}} d t+\int_{0}^{\delta}\left\langle\partial_{x} I(u v), I u\right\rangle_{L^{2}} d t+\frac{1}{2} \int_{0}^{\delta}\left\langle\partial_{x} I v^{2}, I u\right\rangle_{L^{2}} d t \\
& -\frac{3}{2} \int_{0}^{\delta}\left\langle\partial_{x} I v^{2}, I v\right\rangle_{L^{2}} d t+\int_{0}^{\delta}\left\langle\partial_{x} I(u v), I v\right\rangle_{L^{2}} d t+\frac{1}{2} \int_{0}^{\delta}\left\langle\partial_{x} I u^{2}, I v\right\rangle_{L^{2}} d t \\
& +\left\langle\partial_{x}(I u)^{2}, I u\right\rangle_{L^{2}}+\left\langle\partial_{x}(I v)^{2}, I v\right\rangle_{L^{2}}+\int_{0}^{\delta}\left(\frac{1}{2} I_{1}+I_{2}+\frac{1}{2} I_{3}+I_{4}\right) d t .
\end{aligned}
$$

Reordenando trminos obtenemos

$$
\begin{aligned}
R(\delta)= & \frac{3}{2} \int_{0}^{\delta}\left\langle\partial_{x}\left[(I u)^{2}-I u^{2}\right], I u\right\rangle_{L^{2}} d t-\int_{0}^{\delta}\left\langle\partial_{x}[I u I v-I(u v)], I u\right\rangle_{L^{2}} d t \\
& -\frac{1}{2} \int_{0}^{\delta}\left\langle\partial_{x}\left[(I v)^{2}-I v^{2}\right], I u\right\rangle_{L^{2}} d t+\frac{3}{2} \int_{0}^{\delta}\left\langle\partial_{x}\left[(I v)^{2}-I v^{2}\right], I v\right\rangle_{L^{2}} d t \\
& -\int_{0}^{\delta}\left\langle\partial_{x}[I u I v-I(u v)], I v\right\rangle_{L^{2}} d t-\frac{1}{2} \int_{0}^{\delta}\left\langle\partial_{x}\left[(I u)^{2}-I u^{2}\right], I v\right\rangle_{L^{2}} d t \\
= & J_{1}+J_{2}+J_{3}+J_{4}+J_{5}+J_{6} .
\end{aligned}
$$


Usando la identidad de Plancherel y la desigualdad de Cauchy-Schwarz conseguimos

$$
\begin{aligned}
\left|J_{1}\right| & \leq \frac{3}{2} \int_{0}^{\delta}\left|\left\langle\mathcal{F}\left(\partial_{x}\left[(I u)^{2}-I u^{2}\right]\right)(\xi), \mathcal{F}(I u)(\xi)\right\rangle_{L^{2}}\right| d t \\
& =\frac{3}{2} \int_{0}^{\delta}\left|\int_{\mathbb{R}} \mathcal{F}\left(\partial_{x}\left[(I u)^{2}-I u^{2}\right]\right)(\xi) \mathcal{F}(I u)(\xi) d \xi\right| d t \\
& \leq \frac{3}{2} \int_{\mathbb{R}^{2}}\left|\chi_{[0, \delta]}(t)\left\langle\tau-\xi^{3}\right\rangle^{2(b-1)} \mathcal{F}\left(\partial_{x}\left[(I u)^{2}-I u^{2}\right]\right)(\xi)\right| \\
& \leq \frac{3}{2}\left(\int_{\mathbb{R}^{2}} \chi_{[0, \delta]}(t)\left\langle\tau-\xi^{3}\right\rangle^{2(b-1)}\left|\mathcal{F}\left(\partial_{x}\left[(I u)^{2}-I u^{2}\right]\right)(\xi)\right|^{2} d \xi d t\right)^{1 / 2}\left\langle\xi^{3}\right\rangle^{2(1-b)} \mathcal{F}(I u)(\xi) \mid d \xi d t \\
& \leq \frac{3}{2}\left\|\chi_{[0, \delta]}\left(\cdot{ }^{2}\right) \partial_{x}\left[(I u)^{2}-I u^{2}\right]\right\| \|_{X_{0, b-1}} \chi_{[0, \delta]}(t)\left\langle\tau-\chi_{[0, \delta]}\left(\cdot{ }^{3}\right\rangle^{2(1-b) I u}|\mathcal{F}(I u)(\xi)|_{X_{0,1-b}} d \xi d t\right)^{1 / 2} \\
& \leq C\left\|\partial_{x}\left[(I u)^{2}-I u^{2}\right]\right\|\left\|_{X_{0, b-1}^{\delta}}\right\| I u \|_{X_{0,1-b}^{\delta}} \cdot
\end{aligned}
$$

donde $\|f\|_{X_{0, b}^{\delta}}=\left\|\chi_{[0, \delta]}(\cdot t) f\right\|_{X_{0, b}}$ para cualquier $f \in X_{0, b}$. Usando la proposicin 3.4, conseguimos

$$
\left|J_{1}\right| \leq C N^{-\alpha}\|I u\|_{X_{s, b}^{\delta}}^{2}\|I u\|_{X_{0,1-b}^{\delta}} \leq C N^{-\alpha}\|I u\|_{X_{s, b}^{\delta}}^{3} .
$$

Tambin

$$
\begin{aligned}
\left|J_{2}\right| & \leq \int_{0}^{\delta}\left|\left\langle\mathcal{F}\left(\partial_{x}[I u I v-I(u v)]\right)(\xi), \mathcal{F}(I u)(\xi)\right\rangle_{L^{2}}\right| d t \\
& \leq \int_{\mathbb{R}^{2}}\left|\chi_{[0, \delta]}(t)\left\langle\tau-\xi^{3}\right\rangle^{2(b-1)} \mathcal{F}\left(\partial_{x}[I u I v-I(u v)]\right)(\xi)\right| \\
& \leq\left\|\chi_{[0, \delta]}(\cdot t) \partial_{x}[I u I v-I(u v)]\right\|_{X_{0, b-1}}\left\|\chi_{[0, \delta]}(\cdot t) I u\right\|_{X_{0,1-b}} \\
& \leq\left\|\partial_{x}[I u I v-I(u v)]\right\|_{X_{0, b-1}^{\delta}}\|I u\|_{X_{0,1-b}^{\delta}} \\
& \leq C N^{-\alpha}\|I u\|_{X_{s, b}^{\delta}}^{2}\|I v\|_{X_{s, b}^{\delta}} \cdot
\end{aligned}
$$

De la misma manera, tenemos

$$
\begin{array}{ll}
\left|J_{3}\right| \leq C N^{-\alpha}\|I u\|_{X_{s, b}^{\delta}}\|I v\|_{X_{s, b}^{\delta}}^{2}, & \left|J_{4}\right| \leq C N^{-\alpha}\|I v\|_{X_{s, b}^{\delta}}^{3}, \\
\left|J_{5}\right| \leq C N^{-\alpha}\|I u\|_{X_{s, b}^{\delta}}^{3}, & \left|J_{6}\right| \leq C N^{-\alpha}\|I u\|_{X_{s, b}^{\delta}}^{2}\|I v\|_{X_{s, b}^{\delta}} .
\end{array}
$$

Usando (5.9), (5.10), (5.11) y (5.12), la identidad (5.8) da la siguiente ley casi conservada,

$$
\begin{aligned}
\|I u(\delta)\|_{L^{2}}^{2}+\|I v(\delta)\|_{L^{2}}^{2} & \leq\left\|I u_{0}\right\|_{L^{2}}^{2}+\left\|I v_{0}\right\|_{L^{2}}^{2}+|R(\delta)| \\
& \leq\left\|I u_{0}\right\|_{L^{2}}^{2}+\left\|I v_{0}\right\|_{L^{2}}^{2}+C N^{-\alpha}\left(\|I u\|_{X_{s, b}^{\delta}}^{3}+\|I u\|_{X_{s, b}^{\delta}}^{2}\|I v\|_{X_{s, b}^{\delta}}\right. \\
& \left.+\|I u\|_{X_{s, b}^{\delta}}\|I v\|_{X_{s, b}^{\delta}}^{2}+\|I v\|_{X_{s, b}^{\delta}}^{3}\right) .
\end{aligned}
$$

Demostracin del teorema 2.2. Para probar el teorema es suficiente demostrar que la solucin local del problema (2.1) se puede extender a $[0, T]$ para $T>0$ arbitrario.

Para facilitar el anlisis, notamos que si $\lambda \in] 0,1[\mathrm{y}$ definimos

$$
\begin{array}{ll}
u^{\lambda}(x, t)=\lambda^{2} u\left(\lambda x, \lambda^{3} t\right), & v^{\lambda}(x, t)=\lambda^{2} v\left(\lambda x, \lambda^{3} t\right), \\
u_{0}^{\lambda}(x)=\lambda^{2} u_{0}(\lambda x), & v_{0}^{\lambda}(x)=\lambda^{2} v_{0}(\lambda x),
\end{array}
$$

entonces, $(u, v)$ resuelve a $(2.1)$ en $[0, T]$ con informacin inicial $\left(u_{0}, v_{0}\right)$ si y solamente si $\left(u^{\lambda}, v^{\lambda}\right)$ resuelve a (2.1) en $\left[0, T / \lambda^{3}\right]$ con informacin inicial $\left(u_{0}^{\lambda}, v_{0}^{\lambda}\right)$. Por lo tanto, extenderemos $\left(u^{\lambda}, v^{\lambda}\right)$ a $\left[0, T / \lambda^{3}\right]$. 
Como $\left\|u_{0}^{\lambda}\right\|_{H^{s}} \leq C \lambda^{\frac{3}{2}+s}\left\|u_{0}\right\|_{H^{s}} \mathrm{y}\left\|u_{0}^{\lambda}\right\|_{H^{s}} \leq C \lambda^{\frac{3}{2}+s}\left\|u_{0}\right\|_{H^{s}}$, por (3.10) tenemos

$$
\left\|I u_{0}^{\lambda}\right\|_{L^{2}} \leq C N^{-s} \lambda^{\frac{3}{2}+s}\left\|u_{0}\right\|_{H^{s}} \quad \text { y } \quad\left\|I v_{0}^{\lambda}\right\|_{L^{2}} \leq C N^{-s} \lambda^{\frac{3}{2}+s}\left\|v_{0}\right\|_{H^{s}},
$$

donde $N=N(T)$ ser elegido despus, sin embargo ahora $\lambda=\lambda(N)$ exigiendo que

$$
C N^{-s} \lambda^{\frac{3}{2}+s}\left\|u_{0}\right\|_{H^{s}}=\sqrt{\frac{\varepsilon_{0}}{2}} \ll 1 \quad \text { y } \quad C N^{-s} \lambda^{\frac{3}{2}+s}\left\|v_{0}\right\|_{H^{s}}=\sqrt{\frac{\varepsilon_{0}}{2}} \ll 1 .
$$

De (5.15) obtenemos $\lambda=C N^{\frac{2 s}{3+2 s}}$ y usando (5.15) en (5.14) obtenemos

$$
\left\|I u_{0}^{\lambda}\right\|_{L^{2}}^{2} \leq\left(C N^{-s} \lambda^{\frac{3}{2}+s}\left\|u_{0}\right\|_{H^{s}}\right)^{2} \lambda^{2} \leq\left(C N^{-s} \lambda^{\frac{3}{2}+s}\left\|u_{0}\right\|_{H^{s}}\right)^{2} \leq \frac{\varepsilon_{0}}{2} \ll 1
$$

$\mathrm{y}$

$$
\left\|I v_{0}^{\lambda}\right\|_{L^{2}} \leq \frac{\varepsilon_{0}}{2} \ll 1
$$

por lo tanto, si elegimos $\varepsilon_{0}$ arbitrariamente pequeo del teorema 5.1 vemos que el problema (2.1) est bien formulado para todo $t \in[0,1]$.

Ahora, la cantidad casi conservada (5.13), los estimados (5.16) y (5.17), y el teorema 5.1 implican

$$
\left\|I u^{\lambda}(1)\right\|_{L^{2}}^{2}+\left\|I v^{\lambda}(1)\right\|_{L^{2}}^{2} \leq \frac{\varepsilon_{0}}{2}+\frac{\varepsilon_{0}}{2}+4 C N^{-\alpha}\left[\frac{\varepsilon_{0}}{2}\left(\frac{\varepsilon_{0}}{2}\right)^{1 / 2}\right] \leq \varepsilon_{0}+C N^{-\alpha} \varepsilon_{0} .
$$

As, podemos repetir este proceso $C^{-1} N^{\alpha}$ veces antes de duplicar el valor de $\left\|I u^{\lambda}(1)\right\|_{L^{2}}^{2}+\left\|I v^{\lambda}(1)\right\|_{L^{2}}^{2}$. Por este proceso podemos extender la solucin al intervalo de tiempo $\left[0, C^{-1} N^{\alpha}\right]$ tomando $C^{-1} N^{\alpha}$ pasos de tiempo de tamao $O(1)$. Como queremos extender la solucin al intervalo de tiempo $\left[0, T / \lambda^{3}\right]$, elegimos $N=N(T)$ tal que $C^{-1} N^{\alpha} \geq T / \lambda^{3}$. Esto es,

$$
N^{\alpha} \geq \frac{C T}{\lambda^{3}}=C T N^{-\frac{6 s}{3+2 s}}
$$

esto es posible si

$$
\alpha \geq-\frac{6 s}{3+2 s}
$$

Si $s$ es tal que $-\frac{6 s}{3+2 s}<2-3 b$, entonces podemos encontrar $\alpha$ que satisface (5.19) y la hiptesis de la proposicin 3.4. Esta ltima desigualdad se satisface con un valor de $b>\frac{1}{2}$ si $-\frac{6 s}{3+2 s}<\frac{3}{4}$, entonces elegimos $\left.s \in\right]-\frac{3}{10}, 0[$. Esto completa la demostracin del teorema 2.2.

\section{Referencias}

[1] Bona, J.; Chen, H. and Saut, J.C. Boussinesq equations and other systems for small-amplitude long waves in nonlinear dispersive media. Part I. Derivation and linear theory. J. Nonlinear Sci. 19, 283-318. (2002).

[2] - Boussinesq equations and other systems for small-amplitude long waves in nonlinear dispersive media. Part II. Nonlinear theory. Nonlinearity 17, 925-952. (2004).

[3] Bona, J. and Smith, R. The initial-value problem for the Korteweg-de Vries equation. Philos. Trans. Royal Soc. London Series A 278, 555-601. (1975).

[4] Bourgain, J. Fourier transform restriction phenomena for certain lattice subsets and applications to nonlinear evolution equations. Geom. and Funct. Anal. 3, 107-156, 209-262. (1993).

[5] Colliander, J.; Keel,M.; Staffilani, G.; Takaoka, H. and Tao, T. Global well-posedness result for KdV in Sobolev spaces of negative index. Elec. J. Diff. Eq. 26, (2001), $1-7$.

[6] Sharp global well-posedness for $K d V$ and modified $K d V$ on $\mathbb{R}$ and $\mathbb{T}$. Jr. Amer. Math. Soc. 16, 705-749. (2003).

[7] Evans, L.C. Partial Differential Equations. Providence, RI : American Mathematical Society, Second Edition (2010).

[8] Iorio, R. On the Cauchy problem for the Benjamin-Ono equation. Comm PDE. 11, 1031-1081. (1986).

[9] $-K d V, B O$ and friends in weighted Sobolev spaces. Lecture Notes in Math. 1450, 104-121. (1990).

[10] Kenig, C.E. Ponce, G. and Vega, L. Well-posedness of the initial value problem for the Kortweg-de Vries equation. J. Amer. Math. Soc. 4, 323-347. (1991).

[11] Well-posedness and scattering results for the generalized Korteweg-de Vries equation via the contraccin principle. Comm. Pure Appl. Math. 46, 527-620, (1993).

[12] - A bilinear estimate with application to tha KdV equation. J. Amer. Math. Soc. 9, 573-603. (1996).

[13] Linares, F. and Ponce, G. Introduction to Nonlinear Dispersive Equations. Springer-Verlag New York. Universitext, 260 p. (2009).

[14] Prez, Z. Problema de valor inicial para un sistema de Boussinesq. Tesis de Magister, PUCP. (2018).

[15] Rueda, D. Estudio local y global de un sistema tipo Korteweg-De Vries-Burger. Tesis de Magister, PUCP. (2012). 